\title{
SESSION 6: Amino acid transport
}

\author{
The Physiological Society Lecture
}

Amino acid transport, nutrient signalling and cell growth.

Peter M Taylor

College of Life Sciences, University of Dundee, DD1 5EH UK

Amino acids are both raw materials and fuel for cellular protein synthesis and hence for growth and development of human tissues and the adult body form. They also exert regulatory control over numerous, diverse aspects of cell function. For example, large neutral amino acids (LNAA) such as

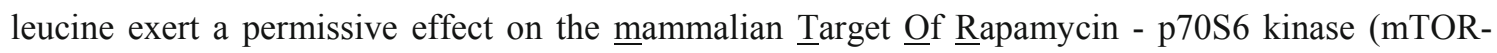
S6K) cell-signalling pathway which promotes net protein synthesis and cell growth. LNAA transporters at the cell surface are therefore potentially limiting components for generation of an anabolic response to LNAA absorbed into the plasma from dietary protein [1].

The System L1 amino acid transporter is the principal LNAA uptake mechanism in many mammalian tissues (including muscle). System L1 is an obligatory LNAA exchanger composed of two protein subunits, a catalytic LNAA permease (either LAT1 or LAT2) and a regulatory glycoprotein (CD98hc; aka 4F2hc). The LAT1-type transporter isoform (SLC7A5) is expressed in many tissues including skeletal muscle, adipose, placenta and brain and is therefore likely to be a major contributor to cellular and whole-body fluxes of LNAA (LAT1 substrates include 6 of the 8 dietary-essential amino acids). LAT1 is able to modulate signalling downstream of intracellular amino acid receptors by regulating intracellular LNAA concentrations through a process of coupled amino acid transport, for example with the ubiquitous SNAT2 (SLC38A2) transporter. Amino acid transporters of the SLC38 family mediate uptake of small neutral amino acids including key intermediary metabolites (e.g. alanine, glutamine and serine) as well as the indispensable amino acids methionine and threonine. This transporter family (classically known as System A) is comprised of three isoforms, SNAT1/2/4, of which SNAT2 is the most widely expressed and extensively regulated member (being responsive to mitogens, amino acid supply, glucocorticoids and cell volume). The SNATs couple the uphill transfer of amino acids to the inward movement of $\mathrm{Na}^{+}$down its electrochemical gradient, hence these transporters develop an outwardly-directed concentration gradient of amino acid substrates which facilitates exchange uptake of a range of LNAA through transporters (such as System L) arranged in parallel with System A in the plasma membrane. This type of coupling (which relies on the availability of a limited group of "shared" System A/L substrates such as glutamine and methionine) appears to be critical for both nutrient uptake and nutrient sensing upstream of mTOR-S6K and may 
therefore have functional importance for control of cell and body growth, as well as in the coupling of growth and survival signals (e.g. from growth factors).

Activation of the mTOR-S6K pathway by LNAA is generally presumed to be directly related to the size of the free intracellular LNAA pool. It is evident that LNAA promote localisation of the mTORC1 protein complex to a subset of endosomalmembranes where Rag-GTPases facilitate interactions between mTOR and its activator, Rheb (see $[1,2]$ for review), although the "LNAA sensing" mechanism itself remains elusive. Nevertheless, whilst some transporters (e.g. LAT1) may function passively in LNAA sensing as conduits for their delivery to the intracellular pool across the plasma membrane, there is a growing body of evidence that suggests that others may function as "transceptors" capable of integrating amino acid sensing/signalling functions with their role as transporters. For example, incubation of cultured mammalian cells with a saturating concentration of the non-metabolisable SNAT2 substrate MeAIB in complete culture medium results in enhanced activation of the mTOR-S6K pathway, despite a substantial reduction in size of the intracellular amino acid pool [3]. This significant depletion of intracellular amino acids arises from inhibited uptake of natural SNAT2 substrates due to competition from the excess of MeAIB, hence reducing their contributionto the "free" intracellular amino acid pool and, in consequence, suppressing the counterdrive for uptake of System L amino acids. This differs from typical "amino acid starvation" studies in that there is no depletion of extracellular amino acids. These observations raise the possibility that amino acid substrate-binding to SNAT2 and/or transport across the membrane may lead to initiation of intracellular signals, as has been shown for a number of transceptors (e.g. Ssy1 and Gap1) in lower eukaryotes (see [1] for review). The signalling molecules linking SNAT2 to mTOR have not been identified in detail, but (i) there is some evidence that SNAT2 may modulate PI3-kinase/PKB signalling upstream of mTOR activation (see [1] for review) and (ii) we have recently identified the protein kinase MLCK II as a putative binding partner for SNAT2 and shown that MLCK inhibition (using ML7) completely blocks adaptive upregulation of SNAT2 in amino acid-deprived rat skeletal muscle cells [3]. Proton-coupled amino acid transporters (PATs) associated with lysosomal membranes have also been identified as elements necessary for supporting the activation of mTORC1 by LNAA [2]. Another intriguing observation from our recent studies is that the depleted intracellular amino acid pool resulting from sustained inhibition of System A with MeAIB remains sufficient to sustain continued cell growth (as measured by increased cell size and protein content), but is apparently insufficient to maintain normal proliferative rate [3]. Activation of the mTOR/S6K signalling axis initiated by amino acid "sensing" through the SNAT2 transceptor may therefore be of particular importance for generating the "anabolic drive" to maintain protein synthetic capacity for cell growth. 


\section{References}

1. Hundal HS, Taylor PM: Amino Acid Transceptors: Gate Keepers Of Nutrient Exchange And Regulators Of Nutrient Signaling. Am J Physiol Endocrinol Metab 2009, 296:E603-E613.

2. Goberdhan DC: Intracellular amino acid sensing and mTORC1-regulated growth: new ways to block an old target? Curr Opin Investig Drugs 2010, 11(12):1360-1367.

3. Pinilla J, Aledo JC, Cwiklinski E, Hyde R, Taylor PM, Hundal HS: SNAT2 transceptor signalling via mTOR: A role in cell growth and proliferation?Front Biosci (Elite Ed) 2011, 3:1289-1299. 


\title{
Modulator proteins that affect the expression and function of the peptide transporter PEPT-1 in C. elegans and mammalian cells
}

\author{
Britta Spanier, Jacqueline Benner and Hannelore Daniel \\ ZIEL Research Center of Nutrition and Food Sciences, Abteilung Biochemie, Technische Universität München, Gregor- \\ Mendel-Str. 2, D-85350 Freising, Germany.
}

Introduction: The intestinal peptide transporter PEPT-1 is a rheogenic $\mathrm{H}^{+}$-dependent carrier responsible for the absorption of di- and tripeptides. Transporter-deficient pept-1 $(\lg 601)$ Caenorhabditis elegans are characterized by impaired growth, development and reproduction and develop obesity [1,2]. The transport function of PEPT-1 is dependent on the membrane potential and on the sodium-proton exchanger NHX-2 for intracellular $\mathrm{pH}$ homeostasis.

Aim: As many membrane proteins are organized in complexes we screened for proteins that directly of indirectly can modulate PEPT-1 expression and transporter function in the nematode.

Methods: 162 genes exclusively expressed in intestinal cells were systematically silenced using an RNAi screen combined with a functional transport assay based on the uptake of the fluorescentlabeled dipeptide $B$-Ala-Lys-AMCA.

Results: Five genes with mammalian homologues were identified that changed PEPT-1 activity and induced a PEPT-1-deficiency phenotype. Silencing of a glutathione peroxidase surprisingly caused an increased PEPT-1 transport function, whereas silencing of NHX-2, of an ER to Golgi cargo transport protein and of two cytosolic peptidases reduced transport associated with lower PEPT-1 protein levels. These proteins seem also to control PEPT1 function assessed in human Caco-2/TC7 cells. In $C$. elegans, treatment with peptidase inhibitors, amino acid supplementation studies and RNAi silencing of the target of rapamycin (TOR) complex two (TORC2, namely rict-1) reduced PEPT-1 function, while RNAi of TORC1 (namely daf-15) increased the B-Ala-Lys-AMCA uptake.

Conclusion: These results provide evidence that intracellular peptide hydrolysis and amino acid levels are part of a sensing system that controls PEPT-1 expression and function and that involves the TOR complexes TORC1 and TORC2.

[1] Meissner, B., Boll, M., Daniel, H., Baumeister, R. Deletion of the intestinal peptide transporter affects insulin and TOR signalling in Caenorhabditis elegans. 2004. J Biol. Chem 279 (35), 3673936745 .

[2] Spanier, B., Lasch, K., Marsch, S., Benner, J., Liao, W., Hu, H., Kienberger, H., Eisenreich, W. and Daniel, H. How the intestinal peptide transporter contributes to an obesity phenotype in Caenorhabditits elegans.2009. PLOS One 4(7):e6279. 


\title{
Structure-function studies on the human proton-coupled amino acid transporter hPAT1 (SLC36A1)
}

\author{
Samyuktha Pillai \& David Meredith
}

Department of Biological \& Medical Science, Faculty of Health \& Life Sciences, Oxford Brookes University, Headington, Oxford OX3 OBP, UK

Introduction: Proteins, as an essential part of our daily nutrition, are broken down to peptides and amino acids prior to absorption in the small intestine. Similarly proteins are hydrolyzed in the lysosomes at an intra-cellular level. In both these cases the breakdown products, amino acids and peptides, are transported across the lipid bilayer by membrane transporters. One such group of transporters is the SLC36 family of Proton-coupled Amino acid Transporters (PATs), which in humans contains four members (based on sequence homology). Two of the SLC36 family members, hPAT1 and hPAT2, have been characterized previously as proton coupled transporters of small neutral amino acids (1). hPAT4 has recently been characterized as a $\mathrm{pH}$ sensitive transporter (optimum $\mathrm{pH}$ 7.4) which is not proton coupled (2). hPAT3 is considered an orphan transporter whose function is unknown. There is currently very little information about the structure of any of the hPAT family members.

Aim: To use membrane prediction software and epitope tagging to elucidate the structure of hPAT1.

Methods: Various trans-membrane domain (TMD) prediction software were used to predict the TMDs of hPAT1. Based on the predictions, the FLAG epitope tag (DYKDDDDK) was inserted in putative extracellular and intracellular regions of hPAT1, using a PCR-based approach. Anti-FLAG HRP-conjugated antibodies were incubated with Xenopus oocytes expressing the FLAG-tagged constructs, and uptake of ${ }^{3} \mathrm{H}$-Proline and luminometry used to give an insight into the membrane topology.

Results: Prediction software suggests hPAT1 has between 9-11 trans-membrane domains. Functional assays showed that no loss of function was observed by insertion of the FLAG tags. From the luminometry studies, hPAT1 appears to have an internal N-terminal, an external C-terminal and 11 TMDs.

Conclusion: The predicted membrane topology of hPAT1 was confirmed by epitope tagging.

\section{References:}

(1) Boll, M., H. Daniel, et al. (2004). The SLC36 family: proton-coupled transporters for the absorption of selected amino acids from extracellular and intracellular proteolysis. Pflügers Archiv European Journal of Physiology 447(5): 776-779.

(2) Pillai SM, Meredith D (2011). SLC36A4 (hPAT4) is a high affinity amino acid transporter when expressed in Xenopus laevis oocytes. J Biol Chem 286(4): 2455-60 